Popout transitions in weakly stretched single-chain multi-block copolymers in poor solvents

This article has been downloaded from IOPscience. Please scroll down to see the full text article.

2011 EPL 9563004

(http://iopscience.iop.org/0295-5075/95/6/63004)

View the table of contents for this issue, or go to the journal homepage for more

Download details:

IP Address: 150.203.177.107

The article was downloaded on 04/10/2011 at 04:55

Please note that terms and conditions apply. 


\title{
Popout transitions in weakly stretched single-chain multi-block copolymers in poor solvents
}

\author{
R. M. Holmes ${ }^{(a)}$ and D. R. M. Williams \\ Department of Applied Mathematics, Research School of Physical Sciences and Engineering, \\ Australian National University - Canberra A.C.T. 0200, Australia
}

received 30 May 2011; accepted in final form 3 August 2011

published online 7 September 2011

PACS 36.20.-r-Macromolecules and polymer molecules

PACS 82.35.Lr - Physical properties of polymers

\begin{abstract}
We show that weakly stretched single-chain multi-block copolymers in poor solvents experience sudden changes in the number of domains when the end-to-end elongation distance of the chain is changed. The simplest symmetric transition of this type, occurring for a fourblock copolymer, is analyzed using self-consistent field theory (SCFT) simulations and a simple free-energy model. The very simple model provides a surprisingly good description of the critical elongation at which the transition occurs as a function of the polymer characteristics.
\end{abstract}

Copyright (c) EPLA, 2011

Introduction. - The stretching of polymers is of fundamental importance in many problems, from the elastic properties of rubber [1] to the behavior of polymeric fluid flows [2]. The entropy properties of polymer chains is what sets them apart from simple liquids and provides them with such rich behavior. The entropic term in the free energy combined with the simpler energetic interaction free energies allows us to describe how a polymer chain deforms under exterior influences. Recent developments in experimental techniques such as atomic force microscope experiments and optical tweezers have allowed tests of these theoretical descriptions of, for example, the behavior of a single polymer chain under an extensional force $[3,4]$. Many such experiments focus on the stretching of various homopolymers, being the simplest case and one for which we have a reasonably complete theoretical description.

The simplest case possible, the stretching of an ideal chain [5], was treated long ago and results in a simple equation for the free energy of an ideal chain of unperturbed length $R_{0}$ stretched a distance $L ; F=k_{B} T \frac{3 L^{2}}{2 R_{0}^{2}}$. From this, equations governing the behavior of ideal chains when confined or elongated in various geometries can be derived. The extension of these descriptions to good solvents has been covered extensively and understanding is now quite good $[6]$.

Single homopolymer chains in poor solvents are more interesting in many ways than homopolymers in good or

\footnotetext{
(a) E-mail: rmholmes@stanford.edu
}

theta solvents, as they undergo some interesting discontinuous transitions. The pioneering work was carried out by Halperin and Zhulina [7] who showed that as extension of a chain in a poor solvent increases, the globule deforms and then undergoes a sudden unwinding transition, forming a ball-and-chain-type conformation. This is a polymeric form of the Rayleigh instability of a liquid crystal. Later work [8] went on to show that at higher extensions there was a further unraveling transition; from a finite-sized ball and chain to a simple extended chain. These studies and others have provided a good understanding of the behavior of homopolymers in poor solvents.

Most polymers are, however, not homopolymers and consist of more than one kind of monomer. With the introduction of a second species of monomer to produce a copolymer, our understanding becomes much more limited. Most studies have focused on strong stretching of di-block and multi-block copolymers in selective solvents, where one species is hydrophobic and one is hydrophilic $[9,10]$. Extensive experimental and some theoretical studies have also been done on the much more complicated case of proteins and DNA [11-13]. However, a blanket understanding of block copolymer stretching does not exist, and there still remain many aspects and parameter ranges yet to be investigated. This paper focuses on one such previously unexplored region of poor-solvent block copolymer single-chain stretching; the regime of weak extension, so that no "ball and chain" Rayleigh instability occurs. For the homopolymer case this was studied early on [7] but the result is somewhat boring. All one gets is deformation 
(a)

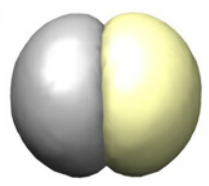

(b)

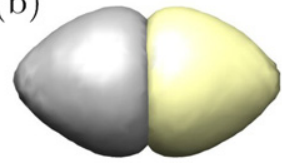

(c)

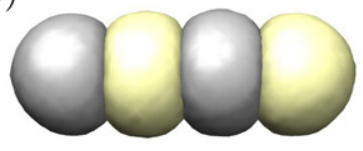

(d)

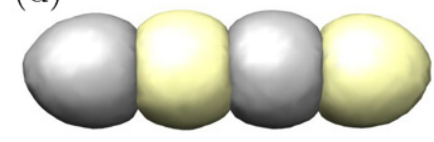

Fig. 1: (Colour on-line) SCFT simulations of a $N=2000$, $\chi_{A B}=0.25, \chi=0.7$ four-block copolymer stretched at different stretching distances (in lattice units) $D=5$ (a), $D=13$ (b), $D=15$ (c) and $D=20$ (d). A clear transition occurs when the formation of four domains is favored over the formation of two domains. For these equilibrium conformations this transition is sharp at a particular stretching distance $D_{t}$, although in real polymers there would be significant hysteresis when passing through $D_{t}[14]$.

from a sphere to an ellipsoid. In marked contrast we shall show that for a block copolymer the physics is in fact interesting.

We investigate the properties of weakly stretched four-block copolymers in poor solvents, where there are two blocks of each species (A and $\mathbf{B}$ ) and all blocks contain the same number of monomers $\frac{N}{4}$. If the polymer is unstretched, then it forms a collapsed globule with two phase-separated domains, one for each species. The domains are approximately hemispheres, but are deformed depending on the relative interaction strengths between polymer-polymer and polymer-solvent [15] (controlled by the Flory interaction parameters $\chi_{A B}$ and $\chi$, respectively). As the polymer is stretched by grabbing both ends, it is forced to adopt a more elongated conformation as the end blocks are pulled away from the center of the conformation. At a particular critical stretching distance $d_{t}$ (in units of the Kuhn length $b$ of the polymer), the loss in entropy means it is no longer favorable for the blocks of the same species to remain together. The blocks are pulled apart and each block forms its own domain in a four-domain conformation. A simulation of this process is shown in fig. 1. This paper presents a brief investigation of this transition and the dependence of the critical elongation $d_{t}$ on the parameters $N, \chi_{A B}$ and $\chi$.

In this work we focus on the simplest possible case where the polymer-solvent interaction strength of each species is the same. While this is not true for the majority of block copolymers, this simple case captures the interesting physics of the transition and would be extendable to the more general cases.

We use a self-consistent field theory (SCFT) simulation method to obtain predictions of the polymer chain conformation with the ends of the chain set apart at varying

distances. For this dense system SCFT is significantly faster than other methods such as Monte Carlo, allowing a broader and more thorough investigation of the parameter space. SCFT has been used extensively for the study of polymer melts $[16,17]$ and minimally for single chains $[15,18]$ and single-chain stretching [19]. The disadvantage of SCFT is that it can only evaluate the equilibrium conformation of the polymer. In a real experiment, there would be significant hysteresis [8] (especially as the polymers are only weakly stretched) when increasing the elongation beyond $d_{t}$ or relaxing the elongation below $d_{t}$.

We first give a brief overview of the SCFT simulation method and its application to stretched single chains. Then a simple free-energy model is developed to predict the critical elongation $d_{t}$ at which the transition from a two-domain to a four-domain globule occurs. The results of the simulations are then presented and analyzed along with the model. Finally we conclude and summarize the outcomes of this work and suggest further research directions.

Self-consistent field theory. - Self-consistent field theory simulations of copolymers involve describing the polymer by density fields $\phi_{A}$ and $\phi_{B}$ on a lattice. These density fields are iteratively updated using partition functions or propagators $Q_{F}$ and $Q_{B}$ that reproduce the Boltzmann distribution and return the connected polymeric nature to the density fields [20].

In this paper we consider a copolymer chain consisting of four alternating blocks of species $\mathbf{A}$ and species $\mathbf{B}$ monomers. Each block is of equal length containing $N / 4$ monomers and the monomers have a Kuhn length $b$. For the SCFT simulations the chain is divided into $T_{s}=500$ steps. Given that the chain begins at $\boldsymbol{r}_{0}$, the forward partition function is denoted $Q_{F}\left(\boldsymbol{r}, \boldsymbol{r}_{0}, s\right)$ and obeys a modified diffusion equation $[21,22]$ :

$$
\frac{\partial}{\partial s} Q_{F}\left(\boldsymbol{r}, \boldsymbol{r}_{0}, s\right)=\frac{b^{2}}{6} \nabla_{\boldsymbol{r}}^{2} Q_{F}\left(\boldsymbol{r}, \boldsymbol{r}_{0}, s\right)-w(\boldsymbol{r}, s) Q_{F}\left(\boldsymbol{r}, \boldsymbol{r}_{0}, s\right) .
$$

Here $w(\boldsymbol{r}, s)$ is the mean potential felt by the $s$-th segment of the chain in the mean-field approximation. The forward partition function has an initial condition $Q_{F}\left(\boldsymbol{r}, \boldsymbol{r}_{0}, T_{s}\right)=\delta\left(\boldsymbol{r}-\boldsymbol{r}_{0}\right)$. Given that the chain ends at the position $\boldsymbol{r}_{T_{s}}$, there is a corresponding backward partition function $Q_{B}\left(\boldsymbol{r}, \boldsymbol{r}_{T_{s}}, s\right)$. This satisfies a similar diffusion equation with initial condition $Q_{B}\left(\boldsymbol{r}, \boldsymbol{r}_{T_{s}}, 0\right)=\delta\left(\boldsymbol{r}-\boldsymbol{r}_{T_{s}}\right)$. Multiplying the forward and backward partition functions and integrating over the lattice gives the total partition function:

$$
Z=\int_{V} Q_{F}\left(\boldsymbol{r}, \boldsymbol{r}_{0}, s\right) Q_{B}\left(\boldsymbol{r}, \boldsymbol{r}_{T_{s}}, s\right) \mathrm{d} \boldsymbol{r} .
$$

The polymer density contribution $\phi_{s}$ of each step along the chain can be obtained from this total partition function:

$$
\phi_{s}(\boldsymbol{r})=\left(\frac{T_{s}}{N} v\right) \frac{Q_{F}\left(\boldsymbol{r}, \boldsymbol{r}_{0}, s\right) Q_{B}\left(\boldsymbol{r}, \boldsymbol{r}_{T_{s}}, s\right)}{Z} .
$$


Here $v=\frac{4}{3} \pi b^{3}$ is the monomer volume. Summing over all step density contributions gives the total densities $\phi_{A}$ and $\phi_{B}$ of species $\mathbf{A}$ and $\mathbf{B}$.

The free energy of a particular polymer conformation contains contributions from the polymer configurational entropy through the partition function $Z$ above, from the energetic interactions between species and from the translational entropy of the solvent. Adopting units of $k_{B} T$, the energetic interaction energies are the polymersolvent interaction energy, described by the Flory interaction parameter $\chi[5]$ :

$$
\left.F_{P S}\right|_{\text {site }}=\chi\left(\phi_{A}+\phi_{B}\right)\left(1-\phi_{A}-\phi_{B}\right)
$$

and the polymer-polymer interaction energy, described by the interaction parameter $\chi_{A B}$ :

$$
\left.F_{A B}\right|_{s i t e}=\chi_{A B} \phi_{A} \phi_{B} .
$$

The free-energy contribution from the translational entropy of the solvent is given by [23]:

$$
\left.F_{S}\right|_{\text {site }}=\left(1-\phi_{A}-\phi_{B}\right) \log \left(1-\phi_{A}-\phi_{B}\right) .
$$

The mean potential $w(\boldsymbol{r}, s)$ used in the diffusion equation is obtained by minimizing these free energy terms with respect to the polymer densities $\phi_{A}$ and $\phi_{B}$. Carrying out this minimization gives for $s$ in an A portion of the chain:

$$
\begin{aligned}
w(\boldsymbol{r}, s)= & w_{A}(\boldsymbol{r})=\chi\left(1-2 \phi_{A}-2 \phi_{B}\right) \\
& +\chi_{A B} \phi_{B}-\log \left(1-\phi_{A}-\phi_{B}\right) .
\end{aligned}
$$

A similar equation exists for $s$ in a $\mathbf{B}$ portion of the chain. An expansion of the $\chi$ term into two sections corresponding to the polymer-solvent interaction of each species $\left(\chi_{A S}\right.$ and $\left.\chi_{B S}\right)$ would allow investigation of the more general case where these interaction strengths are different.

The simulations are conducted on a three-dimensional lattice box of size $51 \times 51 \times 51$ units and we choose the Kuhn length $b$ such that the total polymer volume $v N$ is one-eightieth of the box volume as this was found to be optimal for minimizing finite lattice size effects and allowing efficient computation. The SCFT simulation method involves starting with initial random density fields $\phi_{A}$ and $\phi_{B}$ and performing three steps iteratively on these fields. Firstly, the mean potential $w(\boldsymbol{r}, s)$ is obtained by solving the above equations. Then the modified diffusion equations are solved to obtain the partition functions $Q_{F}$ and $Q_{B}$ [18]. These partition functions are then used to obtain new density fields $\phi_{A}$ and $\phi_{B}$ to be used in the next iteration. After sufficient iteration (we ran each simulation for 25 iterations), the density fields approach fixed fields describing the predicted polymer conformation. More detailed information on the implementation of SCFT used here can be found in a previous paper [18].

We wish to consider stretched copolymer chains where the ends of the chains are fixed a distance $2 D$ from each other ( $D$ is measured in lattice units, while $d=D / b$ is measured in Kuhn lengths). This could be accomplished simply by setting $\boldsymbol{r}_{0}=(0,0,-D)$ and $\boldsymbol{r}_{T_{s}}=(0,0, D)$. However, the delta-function initial conditions on the partition functions create problems in the numerical calculations, so the initial conditions are smoothed over nearby lattice sites by initializing each partition function as a Gaussian centered around the appropriate point. The standard deviation of the Gaussian was chosen as around 1.5 lattice units, as this optimized numerical performance while maintaining the spread as small as possible. This Gaussian spread also allows the stretching distance to vary in smaller steps than a lattice spacing. The centers of the Gaussians can be located in between grid points but sampled at the grid points, giving a non-integer stretching distance $D$. This potentially allows measurements of the critical elongation distance $D_{t}$ to an uncertainty better than the grid spacing.

Figure 1 shows a four-block copolymer simulated using the SCFT method at several stretching distances. With respect to the absolute equilibrium conformations, the clear transition occurs sharply at some distance $D_{t}$. Every simulation conducted around this distance $D_{t}$ gives either the two-domain conformation in fig. 1(b) or the fourdomain conformation in fig. 1(c). However, close to $D_{t}$ the free-energy difference between the two conformations is small so, depending on the random initial conditions, the simulation can get stuck in the wrong one. Therefore, there is a statistical uncertainty in determining the true transition distance even using an infinite number of simulations conducted around $D_{t}$. To determine $D_{t}$ to a reasonable uncertainty, for each polymer parameter set we conducted nine simulations at different distances $D$ chosen algorithmically starting at a guess distance (obtained from the model described in the next section). This algorithm was able to determine $D_{t}$ to within $\pm b$ uncertainty (standard deviation assuming a normal distribution) in most cases and within $\pm 2.5 b$ uncertainty ( $~ 5 \%$ of the total stretching distance) in all cases in which the simulations successfully converged. The simulations contain none of the fluctuations present in real polymer systems and hence the uncertainties above would likely be smaller than uncertainties in the real system. Hysteresis in the real system would also cause the critical elongation to be markedly different from that presented here, although if the polymers were left to equilibrate for long enough, this should not affect the result.

For many extreme ranges of parameters, the simulations would not converge successfully, giving an incorrect value for $D_{t}$. Therefore, strict simulation criteria were developed to identify and eliminate incorrect results. Figure 2 shows plots of the the $N-\chi_{A B}$ and $\chi_{A B^{-}} \chi$ parameter spaces displaying simulation sets passing these criteria with blue circles, and invalid results in red. If $\chi_{A B}$ were too small, sufficient phase separation did not occur in less than 25 iterations. A mixing parameter $M=4 \int \phi_{A} \phi_{B} \mathrm{~d} \boldsymbol{r} / \int\left(\phi_{A}+\right.$ $\left.\phi_{B}\right)^{2} \mathrm{~d} \boldsymbol{r}$ measured the degree of phase separation and if more than one simulation in a particular set of nine had 

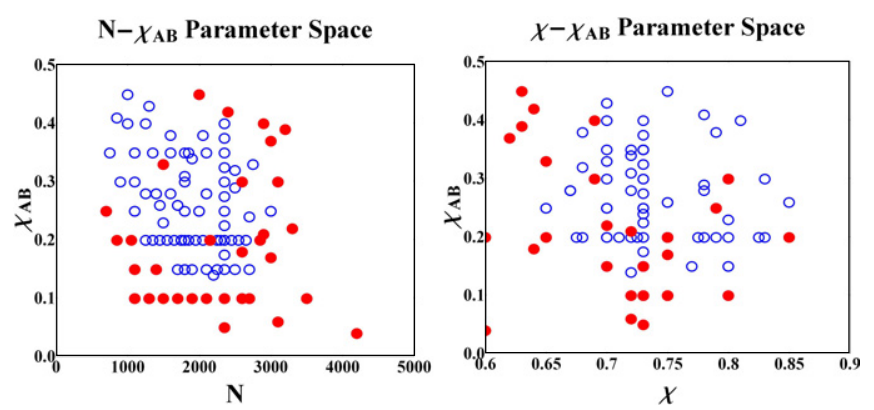

Fig. 2: (Color on-line) Plots of the $N-\chi_{A B}$ and $\chi_{A B}-\chi$ parameter spaces with successful simulation sets locating a valid critical elongation distance $d_{t}$ (open blue circles) and invalid simulation sets not meeting convergence criteria (closed red circles).

$M>0.3$ in the final iterations, this set was judged as invalid (this occurs for many small $\chi_{A B}$ sets in fig. 2). Similarly, if $\chi$ were too small then the globule collapsed slowly; this was indicated by a small standard deviation in the density fields and simulation sets with more than two simulations having a standard deviation of less than 0.038 in the final iterations were judged as invalid. Alternatively, if $\chi_{A B}, \chi$ or $N$ were too large, numerical errors developed and the simulation crashed. Simulation sets where more than two simulations crashed in less than 20 iterations were judged as invalid.

Free-energy model. - A simple free-energy model can be derived to predict the critical elongation $D_{t}$ for 4-block weakly stretched copolymers. We model the stretched polymer as forming a cylinder of length $2 D$ and radius $R$, with the ends of the polymer located at either end of the cylinder. The cylinder is split into two or four sections (of length $D$ or $\frac{D}{2}$, respectively), corresponding to the twodomain or four-domain polymer conformations on each side of the transition. As the outer polymer-solvent surface area is the same for both two- and four-section cylinders, we can ignore the polymer-solvent surface tension freeenergy contribution, only considering A-B surface tension and stretching.

We assume that the poor-solvent interaction causes the polymer to collapse into a globule with some constant volume fraction $\phi$. Given a Kuhn length $b$ and $N$ total monomers in the chain, the radius of the cylinder is determined by

$$
2 D \pi R^{2} \phi=\frac{4}{3} \pi b^{3} N \quad \Longrightarrow \quad R^{2}=\frac{2 b^{3} N}{3 D \phi} .
$$

The surface tension free-energy contribution (we adopt units of $k_{B} T$ ) is simply proportional to the amount of A-B contact area. The exact formula was derived by Helfand and Tagami [24] and gives a total surface tension free energy as a function of the number of sections in the cylinder $S$ :

$$
F_{A B}=\frac{3}{4 \sqrt{6}} \chi_{A B}^{1 / 2} \phi(S-1) \frac{R^{2}}{b^{2}}=\frac{\chi_{A B}^{1 / 2}(S-1) b N}{2 \sqrt{6} D} .
$$

For the entropic stretching free-energy contribution, we take a simplified view where each block stretches like an ideal chain within each cylinder section. As there are four blocks, the four-section case is simple, as each block stretches linearly the length of its section a distance $\frac{D}{2}$. Using the ideal chain stretching equation given in the introduction gives a four-section stretching energy (units of $\left.k_{B} T\right)$ :

$$
F_{S 4}=\frac{6 D^{2}}{N b^{2}}
$$

For the two-section case, the two end blocks stretch the length of their sections a distance $D$. The two middle blocks have a more complicated structure, and we approximate their stretching by assuming each half-block stretches as if it was grafted to the $\mathbf{A}$-B interface at the center of the cylinder. The $\mathbf{A}-\mathbf{B}$ interface area is $\pi R^{2}$, and there are three block junction points on this surface meaning an area per junction point of $\pi R^{2} / 3$. Each half-block stretches in a small cylinder with this cross-sectional area where the height of the small cylinder $H$ is determined by volume conservation given a polymer volume fraction $\phi ; H=\frac{b^{3} N}{2 R^{2} \phi}$. Adding up the contribution of each block gives a two-section stretching energy:

$$
F_{S 2}=\frac{39 D^{2}}{N b^{2}}
$$

The exact values of these stretching free-energy terms are only approximate and we make the assumption that they are accurate up to a pre-multiplying constant $C$. Thus, the total free energy for comparison purposes is $F_{T}=F_{A B}+C F_{S}$, where the parameter $C$ is determined by comparing the model to the results of the SCFT simulations. Setting the free energies of the two- and foursection cylinders equal gives the transition distance $D_{t}$. Converting to distance units of $b$, the critical elongation $d_{t}$ is:

$$
d_{t}=(33 \sqrt{6} C)^{-1 / 3} \chi_{A B}^{1 / 6} N^{2 / 3}
$$

Critical elongation analysis. - Figure 3 plots the critical elongation distance $d_{t}$ obtained from the SCFT simulations against eq. (1) from the cylinder model. The fitting parameter $C$ was chosen as 2.24, indicating that the stretching energy was underestimated by a factor of 2. Despite the simplicity of the model, it appears to work quite well as the data points lie close to the one-to-one line, with a standard deviation from this line of $1.48 \mathrm{Kuhn}$ lengths. This is somewhat surprising given the simplicity of the model and the approximations it makes.

The most important approximation made in the theoretical model is the assumption that the outer surface area of the globule is the same for the two- and fourdomain conformations at the same $D$. Figure 1 shows that the outer shape of the polymer in fact changes significantly with the transition, and that the polymer-solvent surface energy term that depends on $\chi$ should be important. The model ignores this term and has no dependence 


\section{Model-SCFT Critical Elongation Comparison}

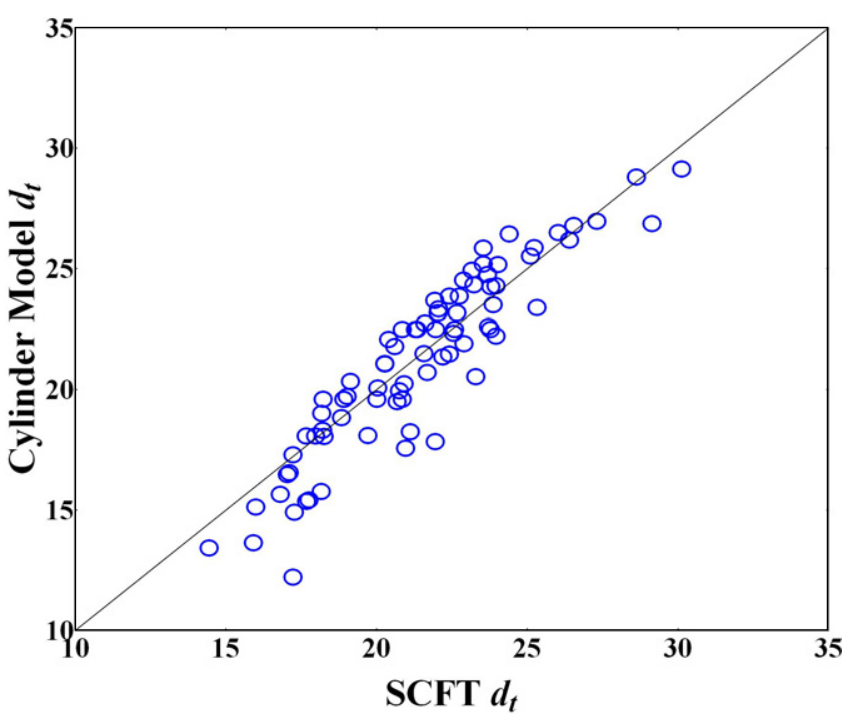

Fig. 3: (Colour on-line) $d_{t}$ obtained from the SCFT simulations against eq. (1) from the cylinder model for the equivalent polymer parameters $N, \chi_{A B}$ and $\chi$. The fitting parameter $C$ is 2.24 . The data describes an approximate one-to-one relationship, with a standard deviation from the one-to-one line of $1.48 b$.

on $\chi$, as the polymer volume fraction $\phi$ cancels out $(\phi$ depends on $\chi[18]$ ). The lower plot in fig. 4 shows the dependence of the critical elongation distance $d_{t}$ on $\chi$ for both the model (constant w.r.t. $\chi$ ) and the simulations. The simulations indicate that $d_{t}$ decreases with $\chi$. This is simply explained because $\chi$ increases the density of the polymer, making the overall globule smaller and the elongation more extreme in relation to the perpendicular globule width. However, the polymer-solvent surface term becomes more important as $\chi$ increases and, therefore, the two-domain conformation, which appears more spherical with a lower polymer-solvent surface area than the fourdomain conformation, is favored by this term. The trend of $d_{t}$ with $\chi$ is therefore weaker than would be expected given a simple shrinking of the globule. The trend of $d_{t}$ with $N$ and $\chi_{A B}$ shown in the other plots in fig. 4 is stronger than the trend with $\chi$ relative to the ranges of the parameters, being one reason why the cylinder model, despite ignoring the effect of $\chi$, describes the system well. Several more complicated models were developed that more correctly described the exterior geometry of the polymer globule and thus included the polymer-solvent energy term. Despite containing an additional fitting parameter controlling the strength of this additional term, these more complicated models resulted in only minor improvements in the spread of fig. 3, indicating that the simple cylinder model captures the essential physics of the problem.

The top plot in fig. 4 shows the dependence of the critical elongation distance $d_{t}$ on the total number of
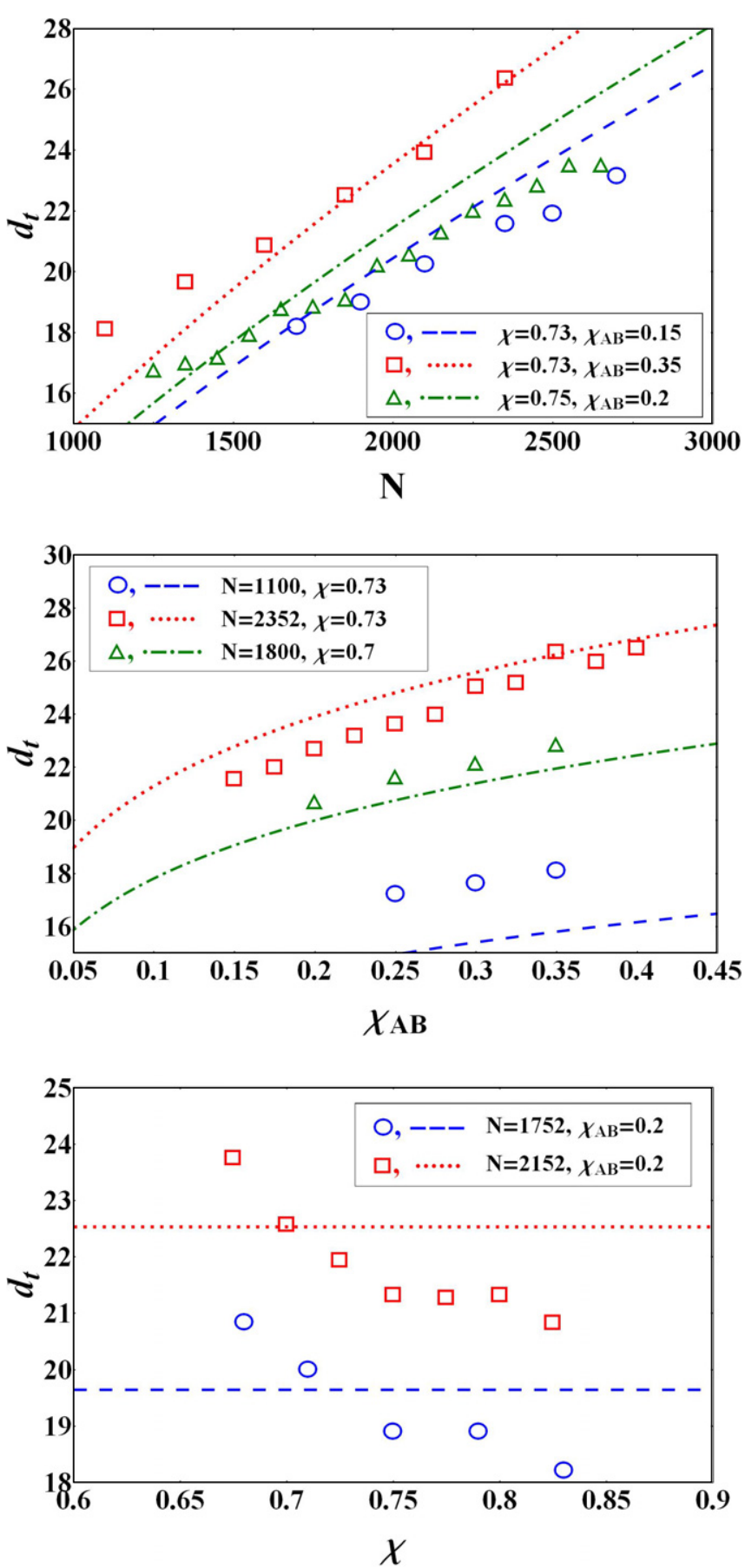

Fig. 4: (Colour on-line) $d_{t}$ as a function of the number of monomers $N$ (top), polymer-polymer interaction strength $\chi_{A B}$ (middle) and polymer-solvent interaction strength $\chi$ (bottom). The data points are from the SCFT simulations and the lines show the model prediction from eq. (1) with fitting parameter $C=2.24$.

monomers $N$ in the chain. $d_{t}$ increases quickly with increasing $N$; the globule gets larger and the elongation becomes less extreme in relation to the perpendicular globule width. The cylinder model appears to overestimate the scaling exponent, predicting a slightly more rapid increase of $d_{t}$ with $N$. In the model, the radius of the 
cylinder increases as $R \sim N^{1 / 2}$ at constant $D$ (for a poor solvent the density is independent of $N$ [23]). A more accurate model of the two-domain conformation, such as a double cone or ellipse, would have a similar semiminor axis $R$ scaling (as in all cases, the volume scales with $R^{2}$ ). However, the surface area of a double cone or ellipse increases more with $R$ than the surface area of a cylinder. Therefore, as $N$ increases, the polymer-solvent surface energy would increasingly favor the four-domain conformation, which is more like a cylinder, over the twodomain conformation. As the model has a cylinder for the two-domain conformation, it overestimates the scaling exponent of $d_{t}$ with $N$, not capturing the polymer-solvent surface energy behavior.

The middle plot in fig. 4 shows the dependence of the critical elongation distance $d_{t}$ on the polymer-polymer interaction strength $\chi_{A B} . d_{t}$ increases with increasing $\chi_{A B}$; the transition from two-domains to four-domains clearly involves an increase in the $\mathbf{A}-\mathbf{B}$ contact area, so as the $\mathbf{A}-\mathbf{B}$ interaction becomes more important the transition is delayed to higher elongations. The trend of $d_{t}$ with $\chi_{A B}$ appears to be modeled quite well, and the constant offsets in this plot are due mainly to the inaccuracies in modeling the trends with the other two parameters.

Conclusions. - This investigation of weakly stretched single-chain multi-block copolymers in poor solvents has shown that these polymers can undergo interesting transitions as the stretching distance increases. A simple cylindrical free-energy model of a four-block copolymer was able to describe the transition that occurs from a twodomain conformation to a four-domain conformation. The critical elongation $d_{t}$ predicted by the cylinder model related to the SCFT prediction with a standard deviation of $1.48 \mathrm{Kuhn}$ lengths, indicating the simple model worked well. The model did not consider the influence of the polymer-solvent interaction, but as the system appeared to have only a small dependence on $\chi$, the model was still able to describe the system satisfactorily. Both the model and simulations only consider equilibrium conformations and in reality there would be significant hysteresis when changing the stretching distance around the critical elongation.

The work presented here would be extendable to more realistic block copolymers where the polymer solvent interaction of each species is different. The small forces and free-energy differences for weakly stretched copolymers made an investigation of the force-elongation curve difficult due to numerical inaccuracies in the SCFT free-energy calculation. If the accuracy of the free-energy calculation in the simulations was improved, this would be an interesting topic for further research. An investigation of higher-order transitions of this type, involving copolymers with more blocks and/or transitions between domain numbers greater than four seems warranted. The physics of strongly stretched chains of this kind also remains to be investigated.

The authors thank the Australian Research Council for supporting this work. The polymer conformation images were produced using the UCSF Chimera package from the Resource for Biocomputing, Visualization, and Informatics at the University of California, San Francisco (supported by NIH P41 RR001081) [14].

\section{REFERENCES}

[1] Treloar R., The Physics of Rubber Elasticity (Clarendon Press, Oxford) 1975.

[2] Bird R., Armstrong R. and Hassager O., Dynamics of Polymeric Liquids, Vol. 1, Fluid Mechanics, 2nd edition (John Wiley and Sons Inc., New York, NY) 1987.

[3] Bemis J., Akhremitchev B. and Walker G., Langmuir, 15 (1999) 2799.

[4] Haupt B., Senden T. and Sevick E., Langmuir, 18 (2002) 2174.

[5] De Gennes P.-G., Scaling Concepts in Polymer Physics (Cornell University Press Ltd.) 1979.

[6] Pincus P., Macromolecules, 9 (1976) 386.

[7] Halperin A. and Zhulina E., Europhys. Lett., 15 (1991) 417.

[8] Cooke I. and Williams D., Europhys. Lett., 64 (2003) 267.

[9] Edwards S. and Williams D., Macromolecules, 38 (2005) 10590.

[10] Lee N.-K., Johner A. and Vilgis T., Macromolecules, 35 (2002) 6043.

[11] Perkins T., Smith D., Larson R. and Chu S., Science, 268 (1995) 83.

[12] Storm C. and Nelson P., Phys. Rev. E, 67 (2003) 051906.

[13] Marko J. and Siggia E., Macromolecules, 28 (1995) 8759.

[14] Pettersen E. et al., J. Comput. Chem., 25 (2004) 1605.

[15] Pinson M. and Williams D., submitted to EPL (2010).

[16] Drolet F. and Fredrickson G., Phys. Rev. Lett., 83 (1999) 4317.

[17] Fredrickson G., Ganesan V. and Drolet F., Macromolecules, 35 (2002) 16.

[18] Holmes R. and Williams D., Macromolecules, 44 (2011) 6172.

[19] Polotsky A. et al., Macromolecules, 42 (2009) 5360.

[20] Matsen M., J. Phys.: Condens. Matter, 14 (2002) R21.

[21] Doi M. and Edwards S., The Theory of Polymer Dynamics (Oxford University Press) 1986.

[22] Helfand E., J. Chem. Phys., 62 (1975) 999.

[23] Williams C., Brochard F. and Frisch H., Annu. Rev. Phys. Chem., 32 (1981) 433.

[24] Helfand E. and Tagami Y., Polym. Lett., 9 (1971) 741. 